\title{
Advances in Simulated Moving Bed Chromatographic Separations
}

Pedro Sá Gomes, Mirjana Minceva, Luis S. Pais, and Alírio E. Rodrigues

\section{1}

Introduction

Introduced by Universal Oil Products (UOP) in the early 1960s by Broughton and Gerhold [1], the Simulated Moving Bed (SMB) appeared predominantly in largescale separations in the petrochemical industry, as a practical implementation of the counter-current continuous chromatographic True Moving Bed (TMB) separation process, avoiding the problems of solid motion by simulating the counter-current bed movement with a synchronous shift of the inlet/outlet ports, while holding the bed immobile. This type of concept is based on the enhancement obtained when a counter-current motion of the stationary phase is applied, leading to solid continous regeneration and recycling, the eluent requirements are reduced and productivity per unit of time and inventory of stationary phase mass is improved. Furthermore, high-purity separation performance can be achieved for stationary phases with low resolution, offering many advantages over elution chromatography and leading to a cleaner, smaller, safer and faster process.

In a so-called TMB separation process, there are two inlet streams, feed and eluent, and two outlet streams, the raffinate and extract, leading to a four-zone delimitation between each inlet streams or collection point. Moreover, there is always present an intrinsic relation between the real SMB unit (Fig. 6.1 a) and the equivalent TMB (Fig. $6.1 \mathrm{~b}$ ), as can be observed.

Recently, SMB technology has found new successful applications as an attractive continuous chromatographic separation process in the biotechnological, pharmaceutical and fine chemistry areas with Novasep equipment in various companies such as UCB Pharma (Belgium) and Aerojet Fine Chemicals (USA). The development of some unconventional SMB strategies, such as the introduction of nonsynchronous inlet/outlet shifts (the Varicol process [2, 3]) variable flux or variable composition in the inlet/outlet streams (PowerFeed $[4,5]$ and Modicon [6, 7], respectively), and also the use of multiple feed or distributed feed [8], have increased the potential of this technique for a wide range of binary separations. 